\title{
Durrmeyer type operators on a simplex
}

\author{
RADU PĂLTĂNEA*
}

ABSTRACT. The paper contains the definition and certain approximation properties of a sequence of Durrmeyer type operators on a simplex, which preserve affine functions and make a link between the multidimensional "genuine" Durrmeyer operators and the multidimensional Bernstein operators.

Keywords: Multidimensional linear positive operators, Durrmeyer type operators, Bernstein operators on a simplex, limit operators, estimates with moduli of continuity.

2020 Mathematics Subject Classification: 41A36, 41A25.

Dedicated to Professor Francesco Altomare, on occasion of his 70th birthday, with esteem and consideration.

\section{INTRODUCTION}

Durrmeyer operators introduced in [10] and independently by Lupaş [17], were one of the most fecund source of inspiration in approximation by positive linear operators. They were be known especially after the paper by Derriennic [7]. In References, we give only a very partial review of contributions in this field. The extension to Jacobi weight was considered by the author in [20], see also [21], [5]. The limit of Durrmeyer operators with Jacobi weight yields the so named "genuine" Durrmeyer operators considered firstly by Chen [6], Goodman and Sharma [14], see also [19], [26], [11]. The eigen-structure of this operators was studied in [22]. For other modifications of Bernstein-Durrmeyer operators mention [18], [27], [16], [3], [1], [15].

In this paper, we are especially interested in the following modification. In [24], there was constructed a family of operators depending on a parameter $\rho$, with property that they preserve linear functions, which make a link between the genuine-Durrmeyer operators and the Bernstein operators in the following mode:

$$
\mathbb{U}_{n}^{\rho}(f)(x)=(1-x)^{n} f(0)+x^{n} f(1)+\sum_{k=0}^{n} \frac{\int_{0}^{1} f(t) t^{k \rho-1}(1-t)^{(n-k) \rho-1} d t}{B(k \rho,(n-k) \rho)} p_{n, k}(x),
$$

where $p_{n, k}(x)=\left(\begin{array}{l}n \\ k\end{array}\right) x^{k}(1-x)^{n-k},(0 \leq k \leq n), f \in C[0,1], x \in[0,1]$. For $\rho=1$, these operators coincide with genuine-Durrmeyer operators and on the other hand $\lim _{\rho \rightarrow \infty} U_{n}^{\rho}=B_{n}$, where $B_{n}$ are the Bernstein operators. These operators are studied more completely in Gonska and the author in [12]. The eigen-structure of operators $U_{n}^{\rho}$ was given in Gonska, Raşa, Stănilă [13].

The extension of Durrmeyer operators on a simplex is very natural. Mention that the first Durrmeyer operators on a simplex were considered by Derriennic [8]. The multidimensional Durrmeyer operators with Jacobi weight were considered by Ditzian [9] and the equivalent of

Received: 17.01.2021; Accepted: 13.02.2021; Published Online: 01.03.2021

*Corresponding author: Radu Păltănea; radupaltanea@yahoo.com

DOI: $10.33205 / \mathrm{cma} .862942$ 
the "genuine"-Durrmeyer operators on a simplex are given by Waldron [28]. The genuine Durrmeyer operators on a simplex preserves affine functions. The generalization of the Durrmeyer operators on a simplex with regard to a arbitrary measure was made by Berdysheva and Jetter [4], see also [25].

The aim of this paper is to extend operators $U_{n}^{\rho}$ on a simplex, obtaining the family of operators $\mathbb{U}_{n}^{\rho}$, which preserve affine functions.

We also construct an additional class of operators $\mathbb{M}_{n}^{\rho, \mathbf{a}}$, depending on a scalar parameter $\rho$ and on a vector parameter a and we prove that operators $\mathbb{U}_{n}^{\rho}$ are the limit of operators $\mathbb{M}_{n}^{\rho, \text { a }}$ when $\mathbf{a} \rightarrow(-1, \ldots,-1)$. This class of operators $\mathbb{M}_{n}^{\rho, \mathbf{a}}$ allows to obtain more simply certain properties of operators $\mathbb{U}_{n}^{\rho}$.

\section{PRELIMINARIES AND DEFINITIONS}

Let $p \in \mathbb{N}$. For any vector $\mathbf{x}=\left(x_{1}, \ldots, x_{p}\right)$, denote $|\mathbf{x}|=x_{1}+\ldots+x_{p}$. For any $p \in \mathbb{R}$, consider the standard simplex in $\mathbb{R}^{p}$.

$$
\Delta_{p}=\left\{\left(x_{1}, \ldots, x_{p}\right)\left|x_{i} \geq 0, \quad\right| \mathbf{x} \mid \leq 1\right\} .
$$

If $g \in C\left(\Delta_{p}\right), p \in \mathbb{N}$, denote by $\int_{\Delta_{p}} g$ the volume integral of $g$ on $\Delta_{p}$.

Fix $m \in \mathbb{N}$. Denote $\mathbf{e}_{k}=(0, \ldots, 0,1,0, \ldots, 0) \in \mathbb{R}^{m},(1 \leq k \leq m)$, where the digit 1 appears at the $k$-th place. Denote also $\mathbf{e}_{0}=(0, \ldots, 0) \in \mathbb{R}^{m}$.

Let $\mathbf{x}=\left(x_{1}, \ldots, x_{m}\right) \in \mathbb{R}^{m}$. Denote the Euclidean norm of $\mathbf{x}$ by $\|\mathbf{x}\|=\sqrt{x_{1}^{2}+\ldots+x_{m}^{2}}$, and the $L_{1}$ norm of $\mathbf{x}$ by $\|\mathbf{x}\|_{1}=\left|x_{1}\right|+\ldots+\left|x_{m}\right|$. If $f \in C\left(\Delta_{m}\right)$, denote $\|f\|=\max _{\mathbf{x} \in \Delta_{m}}|f(\mathbf{x})|$.

For vectors $\mathbf{v}_{0}, \ldots, \mathbf{v}_{p} \in \mathbb{R}^{m}$, denote

$$
\Delta_{\left[\mathbf{v}_{0}, \ldots, \mathbf{v}_{p}\right]}=\left\{\sum_{i=0}^{p} t_{i} \mathbf{v}_{i} \mid t_{0}, \ldots, t_{p} \geq 0, t_{0}+\ldots t_{p}=1\right\},
$$

the simplex with vertices $\mathbf{v}_{0}, \ldots, \mathbf{v}_{p}$. Numbers $t_{0}, \ldots, t_{p}$ are the barycenter coordinates of a point in $\Delta_{\left[\mathbf{v}_{0}, \ldots, \mathbf{v}_{r}\right]}$. Note that $\Delta_{m}=\Delta_{\left[\mathbf{e}_{0}, \ldots, \mathbf{e}_{m}\right]}$.

Fix also a number $n \in \mathbb{N}$. Put

$$
\Lambda=\left\{\mathbf{k}=\left(k_{0}, \ldots, k_{m}\right)|\mathbf{k} \geq 0,| \mathbf{k} \mid=n\right\} .
$$

For $\mathbf{k} \in \Lambda$, denote $\operatorname{supp} \mathbf{k}:=\left\{i \in\{0,1, \ldots, m\} \mid k_{i}>0\right\}$. If supp $\mathbf{k}=\left\{i_{0}<\ldots<i_{p}\right\}$, define $D_{\mathbf{k}}=\Delta_{\left[\mathbf{e}_{i_{0}}, \ldots, \mathbf{e}_{i_{p}}\right]}$.

If $g \in C\left(D_{\mathbf{k}}\right)$, denote by $\int_{D_{k}} g d \sigma$ the integral of $g$ on $D_{k}$. In the case when $D_{\mathbf{k}}=\Delta_{m}$ ， $\int_{D_{k}} g d \sigma=\int_{\Delta_{m}} g$. If $g \in C\left(\Delta_{m}\right)$, then the restriction of $g$ to $D_{\mathbf{k}}$ is denoted also by $g$.

For $\mathbf{k} \in \Lambda$, with supp $\mathbf{k}=\left\{i_{0}<\ldots<i_{p}\right\}$ consider function $\theta_{\mathbf{k}}: \Delta_{p} \rightarrow D_{\mathbf{k}}$ defined by

$$
\theta_{\mathbf{k}}\left(x_{i_{1}}, \ldots, x_{i_{p}}\right)=\sum_{s=1}^{p} x_{i_{s}} \mathbf{e}_{i_{s}}+\left(1-\sum_{s=1}^{p} x_{i_{s}}\right) \mathbf{e}_{i_{0}},\left(x_{i_{1}}, \ldots, x_{i_{p}}\right) \in \Delta_{p} .
$$

Lemma 2.1. Let $\mathbf{k} \in \Lambda$, with $\operatorname{supp} \mathbf{k}=\left\{i_{0}<\ldots<i_{p}\right\}$.

i) If $i_{0}=0$, then

$$
\int_{D_{\mathbf{k}}} g d \sigma=\int_{\Delta_{p}} g \circ \theta_{\mathbf{k}}, g \in C\left(D_{\mathbf{k}}\right)
$$

ii) If $i_{0}>0$, then

$$
\int_{D_{\mathbf{k}}} g d \sigma=\sqrt{p+1} \int_{\Delta_{p}} g \circ \theta_{\mathbf{k}}, g \in C\left(D_{\mathbf{k}}\right) .
$$


Proof. Let prove ii). We have $\theta\left(\Delta_{p}\right)=D_{\mathbf{k}}$. We can write $\theta_{\mathbf{k}}\left(x_{i_{1}}, \ldots, x_{i_{p}}\right)=\mathbf{e}_{i_{0}}+\sum_{s=1}^{p} x_{i_{s}}\left(\mathbf{e}_{i s}-\right.$ $\mathbf{e}_{i_{0}}$ ). Then $\frac{\partial \theta_{\mathbf{k}}}{\partial x_{i_{s}}}=\mathbf{e}_{i_{s}}-\mathbf{e}_{i_{0}}$. Hence

$$
\operatorname{det}\left[\partial \theta_{\mathbf{k}} \cdot\left(\partial \theta_{\mathbf{k}}\right)^{T}\right]:=\operatorname{det}\left[\left\langle\frac{\partial \theta_{\mathbf{k}}}{\partial x_{i_{s}}}, \frac{\partial \theta_{\mathbf{k}}}{\partial x_{i_{t}}}\right\rangle\right]_{1 \leq s, t \leq p}=\operatorname{det}\left(\begin{array}{cccc}
2 & 1 & \ldots & 1 \\
1 & 2 & \ldots & 1 \\
\vdots & \vdots & \ddots & \vdots \\
1 & 1 & \ldots & 2
\end{array}\right)=p+1
$$

Then,

$$
\int_{D_{\mathbf{k}}} g d \sigma=\int_{\Delta_{p}}\left(g \circ \theta_{\mathbf{k}}\right) \sqrt{\operatorname{det}\left[\partial \theta_{\mathbf{k}} \cdot\left(\partial \theta_{\mathbf{k}}\right)^{T}\right]}=\sqrt{p+1} \int_{\Delta_{p}} g \circ \theta_{\mathbf{k}} .
$$

Using the same method, point i) is immediate.

Let $\mathbf{k}=\left(k_{0}, \ldots, k_{m}\right) \in \Lambda$. For $\mathbf{x}=\left(x_{1}, \ldots, x_{m}\right) \in \Delta_{m}$, denote

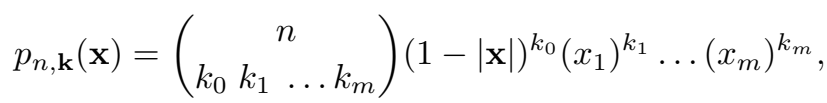

where

$$
\left(\begin{array}{c}
n \\
k_{0} k_{1} \ldots k_{m}
\end{array}\right)=\frac{n !}{k_{0} ! k_{1} ! \ldots k_{m} !} .
$$

The Bernstein operators on the simplex $\Delta_{m}$ are given by

$$
\mathbb{B}_{n}(f)(\mathbf{x})=\sum_{\mathbf{k} \in \Lambda} f\left(\frac{\mathbf{k}}{n}\right) p_{n, \mathbf{k}}(\mathbf{x}), f \in C\left(\Delta_{m}\right), \mathbf{x} \in \Delta_{m} .
$$

Fix a number $\rho>0$. For $\mathbf{k} \in \Lambda$ such that $\operatorname{supp} \mathbf{k}=\left\{i_{0}, \ldots, i_{p}\right\}$ consider function $Q_{\mathbf{k}}^{\rho}: D_{\mathbf{k}} \rightarrow \mathbb{R}$ defined by

$$
Q_{\mathbf{k}}^{\rho}\left(\sum_{s=0}^{p} t_{s} \mathbf{e}_{i_{s}}\right)=\prod_{s=0}^{p} t_{s}^{k_{i_{s}} \rho-1}, \sum_{s=0}^{p} t_{s} \mathbf{e}_{i_{s}} \in D_{\mathbf{k}} .
$$

For $\beta=\left(\beta_{0}, \ldots, \beta_{p}\right), b_{0}, \ldots, b_{p}>0$, consider multidimensional beta function

$$
B(\beta)=\frac{\Gamma\left(\beta_{0}\right) \ldots \Gamma\left(\beta_{p}\right)}{\Gamma(|\beta|)},
$$

where $\Gamma$ is gamma function. If $p=0$, then $B(\beta)=1$.

Let $\mathbf{k} \in \Lambda$, supp $\mathbf{k}=\left\{i_{0}<\ldots<i_{p}\right\}$. From relation (2.5) and relations (2.2) and (2.3), it follows that

$$
\begin{aligned}
& \int_{D_{\mathbf{k}}} Q_{\mathbf{k}}^{\rho} d \sigma=B\left(k_{i_{0}} \rho, \ldots, k_{i_{p}} \rho\right), \text { if } i_{0}=0 \\
& \int_{D_{\mathbf{k}}} Q_{\mathbf{k}}^{\rho} d \sigma=\sqrt{p+1} B\left(k_{i_{0}} \rho, \ldots, k_{i_{p}} \rho\right), \text { if } i_{0}>0 .
\end{aligned}
$$

Definition 2.1. Operators $\mathbb{U}_{n}^{\rho}: C\left(\Delta_{m}\right) \rightarrow C\left(\Delta_{m}\right)$ are defined by

$$
\mathbb{U}_{n}^{\rho}(f)(\mathbf{x})=\sum_{\mathbf{k} \in \Lambda} F_{n, \mathbf{k}}^{\rho}(f) p_{n, \mathbf{k}}(\mathbf{x}), f \in C\left(\Delta_{m}\right), \mathbf{x} \in \Delta_{m},
$$

where

$$
F_{n, \mathbf{k}}^{\rho}(f)=\frac{\int_{D_{\mathbf{k}}} f Q_{\mathbf{k}}^{\rho} d \sigma}{\int_{D_{\mathbf{k}}} Q_{\mathbf{k}}^{\rho} d \sigma}, \mathbf{k} \in \Lambda, f \in C\left(\Delta_{m}\right) .
$$

Remark 2.1. For $\rho=1$, operators $\mathbb{U}_{n}^{\rho}$ coincide with operators constructed by Waldron [28]. 
Definition 2.2. For a vector $\mathbf{a}=\left(a_{0}, \ldots, a_{m}\right)$, with $a_{i}>-1,(0 \leq i \leq m), \rho \geq 1$ and $n \in \mathbb{N}$ define

$$
\mathbb{M}_{n}^{\rho, \mathbf{a}}(f, \mathbf{x})=\sum_{\mathbf{k} \in \Lambda} F_{n, \mathbf{k}}^{\rho, \mathbf{a}}(f) p_{n, \mathbf{k}}(\mathbf{x}), f \in C\left(\Delta_{m}\right), \mathbf{x} \in \Delta_{m}
$$

where

$$
F_{n, \mathbf{k}}^{\rho, \mathbf{a}}(f)=\frac{\int_{\Delta_{m}} f P_{\mathbf{k}}^{\rho, \mathbf{a}}}{\int_{\Delta_{m}} P_{\mathbf{k}}^{\rho, \mathbf{a}}}, f \in C\left(\Delta_{m}\right), \mathbf{k} \in \Lambda
$$

and

$$
P_{\mathbf{k}}^{\rho, \mathbf{a}}(\mathbf{x})=\prod_{s=0}^{m} x_{s}^{k_{s} \rho+a_{s}}, \mathbf{x}=\left(x_{1}, \ldots, x_{m}\right) \in \Delta_{m}, x_{0}=1-|\mathbf{x}|
$$

\section{LINK PROPERTIES}

Theorem 3.1. For any $\rho \geq 1, n \in \mathbb{N}$ and $f \in C\left(\Delta_{m}\right)$, we have

$$
\lim _{\mathbf{a} \rightarrow-\mathbf{1}} \mathbb{M}_{n}^{\rho, \mathbf{a}}(f)(\mathbf{x})=\mathbb{U}_{n}^{\rho}(f)(\mathbf{x}) \text {, uniformly for } \mathbf{x} \in \Delta_{m},
$$

where $-\mathbf{1}=(-1, \ldots,-1) \in \mathbb{N}^{m+1}$.

Proof. It is sufficient to show that

$$
\lim _{\mathbf{a} \rightarrow-\mathbf{1}} F_{n, \mathbf{k}}^{\rho, \mathbf{a}}(f)=F_{n, \mathbf{k}}^{\rho}(f), \mathbf{k} \in \Lambda, f \in C\left(\Delta_{m}\right) .
$$

If supp $\mathbf{k}=\{0,1, \ldots, m\}$, then it is possible to pass to limit $\mathbf{a} \rightarrow \mathbf{- 1}$ by simple replacement $\rho=-1$, because

$$
\lim _{\mathbf{a} \rightarrow-\mathbf{1}} F_{n, \mathbf{k}}^{\rho, \mathbf{a}}(f)=\frac{\int_{\Delta_{m}}\left(f Q_{\mathbf{k}}^{\rho}\right) \circ \theta_{\mathbf{k}}}{\int_{\Delta_{m}} Q_{\mathbf{k}}^{\rho} \circ \theta_{\mathbf{k}}}=\frac{\int_{D_{\mathbf{k}}} f Q_{\mathbf{k}}^{\rho} d \sigma}{\int_{D_{\mathbf{k}}} Q_{\mathbf{k}}^{\rho} d \sigma}
$$

and these integrals exist.

In the sequel, we consider that $\operatorname{supp} \mathbf{k}=\left\{i_{0}<\ldots<i_{p}\right\} \subset\{0,1, \ldots, m\}$, with $p<m$. Also, we denote $\left\{i_{p+1}, \ldots, i_{m}\right\}:=\{0,1, \ldots, m\} \backslash \operatorname{supp} \mathbf{k}$.

If $p=0$, then $D_{\mathbf{k}}=\left\{\mathbf{e}_{i_{0}}\right\}$ and $\pi_{\mathbf{k}}(\mathbf{x})=\mathbf{e}_{i_{0}}, \mathbf{x} \in \Delta_{m}$. Then $\frac{\int_{\Delta_{m}}\left(f \circ \pi_{\mathbf{k}}\right) P_{\mathbf{k}}^{\rho, \mathbf{a}}}{\int_{\Delta_{m}} P_{\mathbf{k}}^{\rho, \mathbf{a}}}=f\left(\mathbf{e}_{i_{0}}\right)$ and on the other hand it follows $F_{n, \mathbf{k}}^{\rho}(f)=\frac{\int_{D_{\mathbf{k}}}\left(f Q_{\mathbf{k}}^{\rho}\right) \circ \theta_{\mathbf{k}}}{\int_{D_{\mathbf{k}}} Q_{\mathbf{k}}^{\rho} \circ \theta_{\mathbf{k}}}=f\left(\mathbf{e}_{i_{0}}\right)$ and (3.13) is clear. We consider now that $p \geq 1$. We have to consider two cases.

Case $1.0 \notin \operatorname{supp} \mathbf{k}$. Then, $0 \in\left\{i_{p+1}, \ldots, i_{m}\right\}$. Consider function $\pi_{\mathbf{k}}: \Delta_{m} \rightarrow D_{\mathbf{k}}$, given by

$$
\pi_{\mathbf{k}}(\mathbf{x})=\sum_{s=1}^{p} x_{i_{s}} \mathbf{e}_{i_{s}}+\left(1-\sum_{s=1}^{p} x_{i_{s}}\right) \mathbf{e}_{i_{0}}, \mathbf{x} \in \Delta_{m} .
$$

Hence $D_{\mathbf{k}}=\Delta_{\left[\mathbf{e}_{i_{0}}, \ldots, \mathbf{e}_{i_{p}}\right]}=\pi\left(\Delta_{m}\right)$. We decompose

$$
F_{n, \mathbf{k}}^{\rho, \mathbf{a}}(f)=\frac{\int_{\Delta_{m}}\left(f \circ \pi_{\mathbf{k}}\right) P_{\mathbf{k}}^{\rho, \mathbf{a}}}{\int_{\Delta_{m}} P_{\mathbf{k}}^{\rho, \mathbf{a}}}+\frac{\int_{\Delta_{m}}\left(f-f \circ \pi_{\mathbf{k}}\right) P_{\mathbf{k}}^{\rho, \mathbf{a}}}{\int_{\Delta_{m}} P_{\mathbf{k}}^{\rho, \mathbf{a}}} .
$$

We show that

$$
\lim _{\mathbf{a} \rightarrow-\mathbf{1}} \frac{\int_{\Delta_{m}}\left(f \circ \pi_{\mathbf{k}}\right) P_{\mathbf{k}}^{\rho, \mathbf{a}}}{\int_{\Delta_{m}} P_{\mathbf{k}}^{\rho, \mathbf{a}}}=F_{n, \mathbf{k}}^{\rho}(f) .
$$


We can write

$$
\begin{aligned}
& \int_{\Delta_{m}}\left(f \circ \pi_{\mathbf{k}}\right) P_{\mathbf{k}}^{\rho, \mathbf{a}} \\
= & \int_{0}^{1} d x_{i_{1}} \int_{0}^{1-x_{i_{1}}} d x_{i_{2}} \ldots \int_{0}^{1-\sum_{s=1}^{p-1} x_{i_{s}}} f\left(\pi_{\mathbf{k}}(\mathbf{x})\right) \prod_{s=1}^{p} x_{i_{s}}^{k_{i_{s}} \rho+a_{i_{s}}} V_{\mathbf{k}}\left(x_{i_{1}}, \ldots, x_{i_{p}}\right) d x_{i_{p}},
\end{aligned}
$$

where

$$
V_{\mathbf{k}}\left(x_{i_{1}}, \ldots, x_{i_{p}}\right)=\int_{0}^{1-\sum_{s=1}^{p} x_{i_{s}}} d x_{i_{p+1}} \ldots \int_{0}^{1-\sum_{s=1}^{m-1} x_{i_{s}}} x_{i_{0}}^{k_{i_{0}} \rho+a_{i_{0}}} \prod_{s=p+1}^{m} x_{i_{s}}^{a_{i_{s}}} d x_{i_{m}},
$$

where $x_{i_{0}}=1-\sum_{s=1}^{m} x_{i_{s}}$. Denote $u=1-\sum_{s=1}^{p} x_{i_{s}}$. Using the change of variables $x_{i_{s}}=u y_{i_{s}}$, $p+1 \leq s \leq m$ one obtains $x_{i_{0}}=u\left(1-\sum_{s=p+1}^{m} y_{i_{s}}\right)$ and then

$$
\begin{aligned}
V_{\mathbf{k}}\left(x_{i_{1}}, \ldots, x_{i_{p}}\right) & =u^{m-p+\sum_{s=p+1}^{m} a_{i_{s}}+a_{i_{0}}+\rho k_{i_{0}}} B\left(\rho k_{i_{0}}+a_{i_{0}}+1, a_{i_{p+1}}+1, \ldots a_{i_{m}}+1\right) \\
& =u^{m-p+\sum_{s=p+1}^{m} a_{i_{s}}+a_{i_{0}}+\rho k_{i_{0}}} \frac{\Gamma\left(k_{i_{0}} \rho+a_{i_{0}}+1\right) \prod_{s=p+1}^{m} \Gamma\left(a_{i_{s}}+1\right)}{\Gamma\left(a_{i_{0}}+\sum_{s=p+1}^{m} a_{i_{s}}+\rho k_{i_{0}}+m-p+1\right)} .
\end{aligned}
$$

We have

$$
\int_{\Delta_{m}} P_{\mathbf{k}}^{\rho, \mathbf{a}}=\frac{\prod_{s=0}^{p} \Gamma\left(k_{i_{s}} \rho+a_{i_{s}}+1\right) \prod_{s=p+1}^{m} \Gamma\left(a_{i_{s}}+1\right)}{\Gamma(|a|+n \rho+m+1)} .
$$

By combining the relations above, we get

$$
\frac{\int_{\Delta_{m}}\left(f \circ \pi_{\mathbf{k}}\right) P_{\mathbf{k}}^{\rho, \mathbf{a}}}{\int_{\Delta_{m}} P_{\mathbf{k}}^{\rho, \mathbf{a}}}=\int_{0}^{1} d x_{i_{1}} \int_{0}^{1-x_{i_{1}}} d x_{i_{2}} \ldots \int_{0}^{1-\sum_{s=1}^{p-1} x_{i_{s}}} f\left(\pi_{\mathbf{k}}(\mathbf{x})\right) T_{\mathbf{k}}^{\mathbf{a}}\left(x_{i_{1}}, \ldots, x_{i_{p}}\right) d x_{i_{p}}
$$

where

$$
\begin{aligned}
T_{\mathbf{k}}^{\mathbf{a}}\left(x_{i_{1}}, \ldots, x_{i_{p}}\right) & =\frac{\Gamma(|\mathbf{a}|+n \rho+m+1)}{\Gamma\left(a_{i_{0}}+\sum_{s=p+1}^{m} a_{i_{s}}+\rho k_{i_{0}}+m-p+1\right) \prod_{s=1}^{p} \Gamma\left(k_{i_{s}} \rho+a_{i_{s}}+1\right)} \\
& \times \prod_{s=1}^{p} x_{i_{s}}^{k_{i_{s}} \rho+a_{i_{s}}}\left(1-\sum_{s=1}^{p} x_{i_{s}}\right)^{m-p+\sum_{s=p+1}^{m} a_{i_{s}}+k_{i_{0}} \rho+a_{i_{0}}}
\end{aligned}
$$

It is possible to pass to limit $\mathbf{a} \rightarrow-\mathbf{1}$ in (3.18) and it follows

$$
\lim _{\mathbf{a} \rightarrow-\mathbf{1}} T_{\mathbf{k}}^{\mathbf{a}}\left(x_{i_{1}}, \ldots, x_{i_{p}}\right)=\frac{\Gamma(n \rho)}{\prod_{s=0}^{p} \Gamma\left(k_{i_{s}} \rho\right)} \prod_{s=1}^{p} x_{i_{s}}^{k_{i_{s}} \rho-1}\left(1-\sum_{s=1}^{p} x_{i_{s}}\right)^{\rho k_{i_{0}}-1} .
$$


By taking into account relations (3.17), (3.19), (2.5), (2.7), (2.3) and then (2.9), we have successively

$$
\begin{aligned}
\lim _{\mathbf{a} \rightarrow-1} \frac{\int_{\Delta_{m}}\left(f \circ \pi_{\mathbf{k}}\right) P_{\mathbf{k}}^{\rho, \mathbf{a}}}{\int_{\Delta_{m}} P_{\mathbf{k}}^{\rho, \mathbf{a}}} & =\frac{\int_{\Delta_{p}}\left(f \cdot Q_{\mathbf{k}}^{\rho}\right) \circ \theta_{\mathbf{k}}}{B\left(k_{i_{0}} \rho, \ldots, k_{i_{p}} \rho\right)} \\
& =\frac{\sqrt{p+1} \int_{\Delta_{p}}\left(f \cdot Q_{\mathbf{k}}^{\rho}\right) \circ \theta_{\mathbf{k}}}{\sqrt{p+1} B\left(k_{i_{0}} \rho, \ldots, k_{i_{p}} \rho\right)} \\
& =\frac{\int_{D_{\mathbf{k}}} f \cdot Q_{\mathbf{k}}^{\rho} d \sigma}{\int_{D_{\mathbf{k}}} Q_{\mathbf{k}}^{\rho} d \sigma} \\
& =F_{n, \mathbf{k}}^{\rho}(f) .
\end{aligned}
$$

So that relation (3.15) was proved. Now, we show that

$$
\lim _{\mathbf{a} \rightarrow-1} \frac{\int_{\Delta_{m}}\left(f-f \circ \pi_{\mathbf{k}}\right) P_{\mathbf{k}}^{\rho, \mathbf{a}}}{\int_{\Delta_{m}} P_{\mathbf{k}}^{\rho, \mathbf{a}}}=0 .
$$

Consider on $\mathbb{R}^{m}$ the norm $\|\mathbf{x}\|_{1}$, defined in the beginning. Let $\varepsilon>0$. There exist $0<\delta<1$, such that if $\mathbf{x} \in \Delta_{m},\left\|\mathbf{x}-\pi_{\mathbf{k}}(\mathbf{x})\right\|<\delta$, then $\left|f(\mathbf{x})-f\left(\pi_{\mathbf{k}}(\mathbf{x})\right)\right|<\varepsilon$. Decompose $\Delta_{m}=A \cup B$, where $A=\left\{\mathbf{x} \in \Delta_{m} \mid\left\|\mathbf{x}-\pi_{\mathbf{k}}(x)\right\|_{1}<\delta\right\}$ and $B=\Delta_{m} \backslash A$. Then

$$
\left|\frac{\int_{A}\left(f-f \circ \pi_{\mathbf{k}}\right) P_{\mathbf{k}}^{\rho, \mathbf{a}}}{\int_{\Delta_{m}} P_{\mathbf{k}}^{\rho, \mathbf{a}}}\right| \leq \varepsilon
$$

Let $\mathrm{x} \in B$. We have

$$
\mathbf{x}-\pi_{\mathbf{k}}(\mathbf{x})=\sum_{j=1}^{m} x_{j} \mathbf{e}_{j}-\sum_{s=1}^{p} x_{i_{s}} \mathbf{e}_{i_{s}}-\left(1-\sum_{s=1}^{p} x_{i_{s}}\right) \mathbf{e}_{i_{0}}=\sum_{s=p+1}^{m} x_{i_{s}} \mathbf{e}_{i_{s}}+\left(-1+\sum_{s=1}^{p} x_{i_{s}}\right) \mathbf{e}_{i_{0}} .
$$

Therefore

$$
\left\|\mathbf{x}-\pi_{\mathbf{k}}(\mathbf{x})\right\|_{1}=\sum_{s=p+1}^{m} x_{i_{s}} \mathbf{e}_{i_{s}}+1-\sum_{s=1}^{p} x_{i_{s}}=x_{0}+2 \sum_{s=p+1}^{m} x_{i_{s}} .
$$

Since $x_{0} \in\left\{i_{p+1}, \ldots, i_{m}\right\}$, it results $\left\|\mathbf{x}-\pi_{\mathbf{k}}(\mathbf{x})\right\|_{1} \leq 3 \sum_{s=p+1}^{m} x_{i_{s}}$. It follows that there is at least an index $j \in\left\{i_{p+1}, \ldots, i_{m}\right\}$, such that $x_{i} \geq \frac{\delta}{3 m}$. Define

$$
B_{j}=\left\{\mathrm{x} \in \Delta_{m} \mid x_{j} \geq \frac{\delta}{3 m}\right\}, j \in\left\{i_{p+1}, \ldots, i_{m}\right\} .
$$

From above, it follows that $B \subset \bigcup_{j \in\left\{0, i_{p+1}, \ldots, i_{m}\right\}} B_{j}$. Therefore

$$
\left|\frac{\int_{B}\left(f-f \circ \pi_{\mathbf{k}}\right) P_{\mathbf{k}}^{\rho, \mathbf{a}}}{\int_{\Delta_{m}} P_{\mathbf{k}}^{\rho, \mathbf{a}}}\right| \leq \frac{2\|f\| \int_{B} P_{\mathbf{k}}^{\rho, \mathbf{a}}}{\int_{\Delta_{m}} P_{\mathbf{k}}^{\rho, \mathbf{a}}} \leq 2\|f\| \sum_{j \in\left\{i_{p+1}, \ldots, i_{m}\right\}} \frac{\int_{B_{j}} P_{\mathbf{k}}^{\rho, \mathbf{a}}}{\int_{\Delta_{m}} P_{\mathbf{k}}^{\rho, \mathbf{a}}} .
$$

We show that

$$
\lim _{\mathbf{a} \rightarrow-1} \frac{\int_{B_{j}} P_{\mathbf{k}}^{\rho, \mathbf{a}}}{\int_{\Delta_{m}} P_{\mathbf{k}}^{\rho, \mathbf{a}}}=0, j \in\left\{i_{p+1}, \ldots, i_{m}\right\} .
$$

We can consider that $a_{j}<0,(0 \leq j \leq m)$. Let $j=i_{r}$, with $p+1 \leq r \leq m$. In integral $\int_{B_{j}} P_{\mathbf{k}}^{\rho, \mathbf{a}}$ if we make the change of variables: $x_{j}=\frac{\delta}{3 m}+\left(1-\frac{\delta}{3 m}\right) y_{j}$ and $x_{\ell}=\left(1-\frac{\delta}{3 m}\right) y_{\ell}$, for 
$\ell \in\{1, \ldots, m\} \backslash\{j\}$. Also $x_{0}=\left(1-\frac{\delta}{3 m}\right) y_{0}$, where $y_{0}=1-\left(y_{1}+\ldots+y_{m}\right)$. Then, we have the equivalence $\left(x_{1}, \ldots, x_{m}\right) \in B_{j} \Longleftrightarrow\left(y_{1}, \ldots, y_{m}\right) \in \Delta_{m}$. We get

$$
\begin{aligned}
& \int_{B_{j}} P_{\mathbf{k}}^{\rho, \mathbf{a}} \\
= & \left(1-\frac{\delta}{3 m}\right)^{m+n \rho+|\mathbf{a}|-a_{i_{r}}} \int_{\Delta_{m}} \prod_{s=0}^{p} y_{i_{s}}^{k_{i_{s}} \rho+a_{i_{s}}} \prod_{s=p+1, s \neq r}^{m} y_{i_{s}}^{a_{i_{s}}}\left(\frac{\delta}{3 m}+\left(1-\frac{\delta}{3 m}\right) y_{i_{r}}\right)^{a_{i_{r}}} .
\end{aligned}
$$

Since $a_{i_{r}}<0$ and $\frac{\delta}{3 m}<1$, we obtain $\left(\frac{\delta}{3 m}+\left(1-\frac{\delta}{3 m}\right) y_{i_{r}}\right)^{a_{i_{r}}} \leq\left(\frac{\delta}{3 m}\right)^{a_{i_{r}}} \leq\left(\frac{\delta}{3 m}\right)^{-1}=\frac{3 m}{\delta}$. Consequently

$$
\begin{aligned}
& \int_{B_{j}} P_{\mathbf{k}}^{\rho, \mathbf{a}} \\
\leq & \frac{3 m}{\delta}\left(1-\frac{\delta}{3 m}\right)^{m+n \rho+|\mathbf{a}|-a_{i_{r}}} \frac{\prod_{s=0}^{p} \Gamma\left(k_{i_{s}} \rho+a_{i_{s}}+1\right) \prod_{s=p+1, s \neq r}^{m} \Gamma\left(a_{i_{s}}+1\right) \Gamma(1)}{\Gamma\left(n \rho+|a|-a_{i_{r}}+m+1\right)} .
\end{aligned}
$$

By taking into account relation (3.16), it results:

$$
\frac{\int_{B_{j}} P_{\mathbf{k}}^{\rho, \mathbf{a}}}{\int_{\Delta_{m}} P_{\mathbf{k}}^{\rho, \mathbf{a}}} \leq \frac{3 m}{\delta}\left(1-\frac{\delta}{3 m}\right)^{m+n \rho+|\mathbf{a}|-a_{i_{r}}} \frac{\Gamma(n \rho+|\mathbf{a}|+m+1)}{\Gamma\left(n \rho+|\mathbf{a}|-a_{i_{r}}+m+1\right) \Gamma\left(a_{i_{r}}+1\right)} .
$$

But

$$
\lim _{\mathbf{a} \rightarrow-\mathbf{1}}\left(1-\frac{\delta}{3 m}\right)^{m+n \rho+|\mathbf{a}|-a_{i_{r}}} \frac{\Gamma(n \rho+|\mathbf{a}|+m+1)}{\Gamma\left(n \rho+|\mathbf{a}|-a_{i_{r}}+m+1\right)}=\left(1-\frac{\delta}{3 m}\right)^{n \rho} \frac{\Gamma(n \rho)}{\Gamma(n \rho+1)}
$$

and $\lim _{\mathbf{a} \rightarrow-\mathbf{1}} \Gamma\left(a_{i_{r}}+1\right)=\infty$. Then, one obtains relation (3.22).

Case 2. $i_{0}=0$. Then supp $\mathbf{k}=\left\{0=i_{0}<i_{1}<\ldots<i_{p}\right\}$, where $0 \leq p \leq m-1$. Define the function $\pi_{\mathbf{k}}: \Delta_{m} \rightarrow D_{\mathbf{k}}$, by

$$
\pi_{\mathbf{k}}(\mathbf{x})=\sum_{s=1}^{p} x_{i_{s}} \mathbf{e}_{i_{s}}, \mathbf{x}=\left(x_{1}, \ldots, x_{m}\right) \in \Delta_{m} .
$$

The method of the proof is similar as in Case 1. Consider the decomposition of the form given in (3.14). First, we show the corresponding relation (3.15). For $\left(x_{i_{1}}, \ldots, x_{i_{p}}\right) \in \Delta_{p}$, we denote

$$
U\left(x_{i_{1}}, \ldots, x_{i_{p}}\right)=\left\{\left(x_{i_{p+1}}, \ldots, x_{i_{m}}\right) \mid x_{i_{s}} \geq 0,(p+1 \leq s \leq m), \sum_{s=p+1}^{m} x_{i_{s}} \leq 1-\sum_{s=1}^{p} x_{i_{s}}\right\} .
$$

Then, we can write

$$
\begin{aligned}
& \int_{\Delta_{m}}\left(f \circ \pi_{\mathbf{k}}\right) \cdot P_{\mathbf{k}}^{\rho, \mathbf{a}} \\
= & \int_{\Delta_{p}} f\left(\sum_{s=1}^{p} x_{i_{s}} \mathbf{e}_{i_{s}}\right) \prod_{s=1}^{p} x_{i_{s}}^{k_{i_{s}} \rho+a_{i_{s}}} d x_{i_{1}} \ldots d x_{i_{p}} \\
\times & \int_{U\left(x_{i_{1}}, \ldots, x_{i_{p}}\right)} \prod_{s=p+1}^{m} x_{i_{s}}^{a_{i_{s}}} x_{0}^{k_{0} \rho+a_{0}} d x_{i_{p+1}} \ldots d x_{i_{m}} .
\end{aligned}
$$


Denote $u=1-x_{i_{1}}-\ldots-x_{i_{p}}$. Using the change of variables $x_{\ell}=u y_{i_{\ell}}, p+1 \leq \ell \leq m$ in the interior integral, we obtain

$$
\begin{aligned}
& \int_{U\left(x_{i_{1}}, \ldots, x_{i_{p}}\right)} \prod_{s=p+1}^{m} x_{i_{s}}^{a_{i_{s}}} x_{0}^{k_{0} \rho+a_{0}} d x_{i_{p+1}} \ldots d x_{i_{m}} \\
= & u^{m-p+a_{0}+a_{i_{p+1}}+\ldots+a_{i_{m}}+k_{0} \rho} B\left(k_{0} \rho+a_{0}+1, a_{i_{p+1}}+1, \ldots, a_{i_{m}}+1\right) .
\end{aligned}
$$

By taking also into account relation (3.16), we obtain

$$
\begin{aligned}
& \frac{\int_{\Delta_{m}}(f \circ \pi) \cdot P_{\mathbf{k}}^{\rho, \mathbf{a}}}{\int_{\Delta_{m}} P_{\mathbf{k}}^{\rho, \mathbf{a}}} \\
= & \int_{\Delta_{p}} f\left(\sum_{s=1}^{p} x_{i_{s}} \mathbf{e}_{i_{s}}\right) \prod_{s=1}^{p} x_{i_{s}}^{k_{i_{s}} \rho+a_{i_{s}}}\left(1-\sum_{s=1}^{p} x_{i_{s}}\right)^{m-p+a_{0}+\sum_{s=p+1}^{m} a_{i_{s}}+k_{0} \rho} d x_{i_{1}} \ldots d x_{i_{p}} \\
\times & \frac{\Gamma(n \rho+|\mathbf{a}|+m+1)}{\Gamma\left(k_{0} \rho+a_{0}+a_{i_{p+1}}+\ldots+a_{i_{m}}+m-p+1\right) \prod_{s=1}^{p} \Gamma\left(k_{i_{s}} \rho+a_{i_{s}}+1\right)} .
\end{aligned}
$$

Using (2.1), (2.6), (2.2) and (2.9), it follows

$$
\lim _{\mathbf{a} \rightarrow \mathbf{1}} \frac{\int_{\Delta_{m}}\left(f \circ \pi_{\mathbf{k}}\right) \cdot P_{\mathbf{k}}^{\rho, \mathbf{a}}}{\int_{\Delta_{m}} P_{\mathbf{k}}^{\rho, \mathbf{a}}}=\frac{\int_{\Delta_{p}}\left(f \cdot Q_{\mathbf{k}}^{\rho}\right) \circ \theta_{\mathbf{k}}}{B\left(k_{i_{0}} \rho, \ldots, k_{i_{p}} \rho\right)}=\frac{\int_{D_{\mathbf{k}}} f \cdot Q_{\mathbf{k}}^{\rho}}{\int_{D_{\mathbf{k}}} Q_{\mathbf{k}}^{\rho}}=F_{n, \mathbf{k}}^{\rho}(f) .
$$

So that relation (3.15) is proved.

In order to prove the corresponding relation (3.20), let $\varepsilon>0$ arbitrarily chosen. There is $0<$ $\delta<1$, such that inequality $\left\|\mathbf{x}-\pi_{\mathbf{k}}(\mathbf{x})\right\|_{1}<\delta, \mathbf{x} \in \Delta_{m}$ implies $\left|f(\mathbf{x})-f\left(\pi_{\mathbf{k}}(x)\right)\right|<\varepsilon$. Consider the sets $A=\left\{\mathbf{x} \in \Delta_{m} \mid\left\|\mathbf{x}-\pi_{\mathbf{k}}(\mathbf{x})\right\|_{1}<\delta\right\}$ and $B=\Delta_{m} \backslash A$. We have

$$
\left|\frac{\int_{\Delta_{m}}\left(f-f \circ \pi_{\mathbf{k}}\right) P_{\mathbf{k}}^{\rho, \mathbf{a}}}{\int_{\Delta_{m}} P_{\mathbf{k}}^{\rho, \mathbf{a}}}\right| \leq \varepsilon+2\|f\| \frac{\int_{B} P_{\mathbf{k}}^{\rho, \mathbf{a}}}{\int_{\Delta_{m}} P_{\mathbf{k}}^{\rho, \mathbf{a}}} .
$$

If $\mathbf{x} \in B$, there is $j \in\{p+1, \ldots, m\}$ such that $x_{j} \geq \frac{\delta}{m}$. Indeed, otherwise we have $\left\|\mathbf{x}-\pi_{\mathbf{k}}(\mathbf{x})\right\|_{1}=$ $x_{i_{p+1}}+\ldots x_{i_{m}}<(m-p) \frac{\delta}{m} \leq \delta$, which is a contradiction. Define

$$
B_{j}:=\left\{\mathbf{x}=\left(x_{1}, \ldots, x_{m}\right) \in \Delta_{m} \mid x_{j} \geq \frac{\delta}{m}\right\}, j \in\left\{i_{p+1}, \ldots, i_{m}\right\} .
$$

Therefore $B \subset \bigcup_{j=p+1}^{m} B_{j}$, which implies

$$
\frac{\int_{B} P_{\mathbf{k}}^{\rho, \mathbf{a}}}{\int_{\Delta_{m}} P_{\mathbf{k}}^{\rho, \mathbf{a}}} \leq \sum_{j=p+1}^{m} \frac{\int_{B_{j}} P_{\mathbf{k}}^{\rho, \mathbf{a}}}{\int_{\Delta_{m}} P_{\mathbf{k}}^{\rho, \mathbf{a}}}
$$

Fix $r \in\{p+1, \ldots, m\}$ and $j=i_{r}$. With the change of variables $x_{i_{r}}=\frac{\delta}{m}+\left(1-\frac{\delta}{m}\right) y_{i_{r}}$ and $x_{\ell}=\left(1-\frac{\delta}{m}\right) y_{\ell}, \ell \in\{1, \ldots, m\} \backslash\{r\}$; the condition $\mathbf{x} \in B_{j}$ is equivalent to $\left(y_{1}, \ldots, y_{m}\right) \in \Delta_{m}$. We obtain $x_{0}=\left(1-\frac{\delta}{m}\right) y_{0}$ and then

$$
\begin{aligned}
& \int_{B_{j}} P_{\mathbf{k}}^{\rho, \mathbf{a}} \\
= & \left(1-\frac{\delta}{m}\right)^{m+n \rho+|\mathbf{a}|-a_{i_{s}}} \int_{\Delta_{m}} \prod_{\ell=0, \ell \neq i_{r}}^{m} y^{k_{\ell} \rho+a_{\ell}}\left(\frac{\delta}{m}+\left(1-\frac{\delta}{m}\right) y_{i_{r}}\right)^{a_{i_{r}}} d y_{1} \ldots d y_{m} .
\end{aligned}
$$

We have $\left(\frac{\delta}{m}+\left(1-\frac{\delta}{m}\right) y_{i_{r}}\right)^{a_{i_{r}}} \leq\left(\frac{\delta}{m}\right)^{a_{i_{r}}}<\left(\frac{\delta}{m}\right)^{-1}=\frac{m}{\delta}$. Then 


$$
\begin{aligned}
& \int_{B_{j}} P_{\mathbf{k}}^{\rho, \mathbf{a}} \leq \frac{m}{\delta}\left(1-\frac{\delta}{m}\right)^{m+n \rho+|\mathbf{a}|-a_{i_{r}}} \\
& \times \frac{\prod_{s=0}^{p} \Gamma\left(k_{i_{s}} \rho+a_{i_{s}}+1\right) \prod_{s=p+1, s \neq r}^{m} \Gamma\left(a_{i_{s}}+1\right) \Gamma(1)}{\Gamma\left(n \rho+|\mathbf{a}|-a_{i_{r}}+m+1\right)} .
\end{aligned}
$$

Using also relation (3.16), it follows

$$
\frac{\int_{B_{j}} P_{\mathbf{k}}^{\rho, \mathbf{a}}}{\int_{\Delta_{m}} P_{\mathbf{k}}^{\rho, \mathbf{a}}} \leq \frac{m}{\delta}\left(1-\frac{\delta}{m}\right)^{m+n \rho+|\mathbf{a}|-a_{i_{r}}} \frac{\Gamma(n \rho+|\mathbf{a}|+m+1)}{\Gamma\left(n \rho+|\mathbf{a}|-a_{j}+m+1\right) \Gamma\left(a_{j}+1\right)} .
$$

But

$$
\lim _{\mathbf{a} \rightarrow \mathbf{1}}\left(1-\frac{\delta}{m}\right)^{m+n \rho+|\mathbf{a}|-a_{i_{r}}} \frac{\Gamma(n \rho+|\mathbf{a}|+m+1)}{\Gamma\left(n \rho+|\mathbf{a}|-a_{j}+m+1\right)}=\left(1-\frac{\delta}{m}\right)^{n \rho} \frac{\Gamma(n \rho)}{\Gamma(n \rho+1)}
$$

and $\lim _{\mathbf{a} \rightarrow \mathbf{1}} \Gamma\left(a_{j}+1\right)=\infty$. Then, the corresponding relations (3.22) are true. Now, it is simple to deduce that (3.20) is valid.

Remark 3.2. In the case $\rho=1$, Theorem 3.1 was proved in [28] but using a method which is not applicable here. In unidimensional case, Theorem 3.1 was proved in [12].

Theorem 3.2. For any $f \in C\left(\Delta_{m}\right)$, we have

$$
\lim _{\rho \rightarrow \infty} \mathbb{U}_{n}^{\rho}(f)(\mathbf{x})=\mathbb{B}_{n}(f)(\mathbf{x}), \text { uniformly for } \mathbf{x} \in \Delta_{m} .
$$

Proof. It is sufficient to show that

$$
\lim _{\rho \rightarrow \infty} F_{n, \mathbf{k}}^{\rho}(f)=f\left(\frac{k_{1}}{n}, \ldots, \frac{k_{m}}{n}\right), \mathbf{k}=\left(k_{0}, k_{1}, \ldots, k_{m}\right) \in \Lambda, f \in C\left(\Delta_{m}\right) .
$$

Let supp $\mathbf{k}=\left\{i_{0}, \ldots, i_{p}\right\} \subset\{0,1, \ldots, m\}, p \geq 0$. If $p=0$, then relation (3.23) is immediate. Now consider that $p \geq 1$. We introduce simplified notations as follows. Denote $\mu_{j}=k_{i_{j}},(0 \leq j \leq p)$. Recall that $D_{\mathbf{k}}=\left\{\sum_{j=0}^{p} y_{j} \mathbf{e}_{i_{j}} \mid y_{j} \geq 0,(0 \leq j \leq p), \sum_{j=0}^{p} y_{j}=1\right\}$. Define $\varphi: \Delta_{p} \rightarrow \mathbb{R}$ by

$$
\varphi(\mathbf{y})=\prod_{j=1}^{p} y_{j}^{\mu_{j}}(1-|\mathbf{y}|)^{\mu_{0}}, \mathbf{y}=\left(y_{1}, \ldots, y_{p}\right) \in \Delta_{p} .
$$

We have $\varphi \geq 0$ on $\Delta_{p}$. Since $\mu_{j} \geq 1,0 \leq j \leq p$, it follows that $\varphi=0$ on the frontier of $\Delta_{p}$. Consequently, the maximum of $\varphi$ is reached in the interior of domain $\Delta_{p}$. It is simple to show that the unique interior critical point of $\varphi$ is $\mathbf{y}^{*}=\left(\frac{\mu_{1}}{n}, \ldots, \frac{\mu_{p}}{n}\right) \in \Delta_{p}$. Then, $\mathbf{y}^{*}$ is the unique maximum point of $\varphi$.

Define $g \in C\left(\Delta_{p}\right), g=f \circ \theta_{\mathbf{k}}$, where $\theta_{\mathbf{k}}$ was defined in (2.1).

Let $\varepsilon>0$ arbitrarily chose. We can choose a number $r>0$, such that $B_{r}\left(\mathbf{y}^{*}\right)=\left\{\mathbf{y} \in \mathbb{R}^{p} \mid \| \mathbf{y}-\right.$ $\left.\mathbf{y}^{*} \|<r\right\} \subset \operatorname{Int} \Delta_{p}$ and $\left|g(\mathbf{y})-g\left(\mathbf{y}^{*}\right)\right|<\frac{\varepsilon}{2}$, for all $\mathbf{y} \in B_{r}\left(\mathbf{y}^{*}\right)$. Define $M=\max \{\varphi(\mathbf{y}) \mid \mathbf{y} \in$ $\left.\overline{\Delta_{p} \backslash B_{r}\left(\mathbf{y}^{*}\right)}\right\}$. Then $M<\varphi\left(\mathbf{y}^{*}\right)$. Choose $M<M_{1}<\varphi\left(\mathbf{y}^{*}\right)$. There is $\delta>0$, such that $0<\delta<r$ and $\varphi(\mathbf{y}) \geq M_{1}$, for all $\mathbf{y} \in B_{\delta}\left(\mathbf{y}^{*}\right)$.

For $\rho>1$, define $\Psi=\Psi_{\rho, \mathbf{k}} \in C\left(\Delta_{p}\right), \Psi=Q_{\mathbf{k}}^{\rho} \circ \theta_{\mathbf{k}}$, where $Q_{\mathbf{k}}^{\rho}$ and $\theta_{\mathbf{k}}$ are defined in (2.5) and (2.1), respectively. Then we can write $\Psi=\varphi^{\rho-1} \cdot \eta$, where

$$
\eta(\mathbf{y})=\prod_{j=1}^{p} y_{j}^{\mu_{j}-1}(1-|\mathbf{y}|)^{\mu_{0}-1}, \mathbf{y}=\left(y_{1}, \ldots, y\right) \in \Delta_{p} .
$$


We have

and

$$
\int_{\Delta_{p} \backslash B_{r}\left(y^{*}\right)} \Psi=\int_{\Delta_{p} \backslash B_{r}\left(y^{*}\right)}(\varphi)^{\rho-1} \eta \leq\|\eta\| M^{\rho-1} \operatorname{vol}\left(\Delta_{p}\right)
$$

$$
\int_{B_{r}\left(y^{*}\right)} \Psi \geq \int_{B_{\delta}\left(y^{*}\right)}(\varphi)^{\rho-1} \eta \geq h \cdot M_{1}^{\rho-1} \operatorname{vol}\left(B_{\delta}\left(\mathbf{y}^{*}\right)\right),
$$

where $h=\min \left\{\eta(\mathbf{y}) \mid \mathbf{y} \in \overline{B_{r}\left(\mathbf{y}^{*}\right)}\right\}>0$. Then

$$
\frac{\int_{\Delta_{p} \backslash B_{r}\left(\mathbf{y}^{*}\right)} \Psi}{\int_{B_{r}\left(y^{*}\right)} \Psi} \leq \frac{\|\eta\| \cdot \operatorname{vol}\left(\Delta_{p}\right)}{h \cdot \operatorname{vol}\left(B_{\delta}\left(\mathbf{y}^{*}\right)\right)}\left(\frac{M}{M_{1}}\right)^{\rho-1} .
$$

It is possible to choose $\rho_{0}>1$, such that

$$
2\|f\| \cdot \frac{\int_{\Delta_{p} \backslash B_{r}\left(\mathbf{y}^{*}\right)} \Psi}{\int_{B_{r}\left(y^{*}\right)} \Psi}<\frac{\varepsilon}{2}, \forall \rho>\rho_{0} .
$$

Using formula (2.2) or formula (2.3) depending on the condition $0 \in \operatorname{supp} \mathbf{k}$ or $0 \notin \operatorname{supp} \mathbf{k}$, in both cases it results

$$
F_{n, \mathbf{k}}^{\rho}(f)=\frac{\int_{D_{\mathbf{k}}} f \cdot Q_{\mathbf{k}}^{\rho} d \sigma}{\int_{D_{\mathbf{k}}} Q_{\mathbf{k}}^{\rho} d \sigma}=\frac{\int_{\Delta_{p}}\left(f \cdot Q_{\mathbf{k}}^{\rho}\right) \circ \theta_{\mathbf{k}}}{\int_{\Delta_{p}} Q_{\mathbf{k}}^{\rho} \circ \theta_{\mathbf{k}}}=\frac{\int_{\Delta_{p}} g \cdot \Psi}{\int_{\Delta_{p}} \Psi} .
$$

Then, for $\rho>\rho_{0}$ :

$$
\begin{aligned}
\left|F_{n, \mathbf{k}}^{\rho}(f)-g\left(\mathbf{y}^{*}\right)\right| & =\left|\frac{\int_{\Delta_{p}} g \cdot \Psi}{\int_{\Delta_{p}} \Psi}-g\left(\mathbf{y}^{*}\right)\right| \\
& \leq \frac{\int_{\Delta_{p}} \mid\left(g-g\left(y^{*}\right) \mid \cdot \Psi\right.}{\int_{\Delta_{p}} \Psi} \\
& \leq \frac{\int_{\Delta_{p} \backslash B_{r}\left(\mathbf{y}^{*}\right)}\left|g-g\left(y^{*}\right)\right| \cdot \Psi}{\int_{\Delta_{p}} \Psi}+\frac{\int_{B_{r}\left(\mathbf{y}^{*}\right)} \mid\left(g-g\left(y^{*}\right) \mid \cdot \Psi\right.}{\int_{\Delta_{p}} \Psi} \\
& <\frac{\varepsilon}{2}+\frac{\varepsilon}{2}=\varepsilon .
\end{aligned}
$$

Finally note that $g\left(\mathbf{y}^{*}\right)=f\left(\frac{k_{1}}{n}, \ldots, \frac{k_{m}}{n}\right)$.

\section{CONVERGENCE PROPERTIES}

The moments of operators play a crucial role in the study of the convergence properties of a sequence of linear positive operators. The computation of moments of operators $\mathbb{M}_{n}^{\rho, \mathbf{a}}$ and $\mathbb{U}_{n}^{\rho}$ can be reduced to the moments of the Bernstein operators $\mathbb{B}_{n}$.

Define the functions $\mathbf{1}_{\Delta_{m}} \in C\left(\Delta_{m}\right), \mathbf{1}_{\Delta_{m}}(\mathbf{x})=1$ and $\operatorname{pr}_{i} \in C\left(\Delta_{m}\right),(1 \leq i \leq m), \mathrm{pr}_{i}(\mathbf{x})=x_{i}$, $\mathbf{x}=\left(x_{1}, \ldots, x_{m}\right) \in \Delta_{m}$.

Define

$$
\|\bullet-\bar{x}\|\left(t_{1}, \ldots, t_{m}\right)=\sqrt{\sum_{i=1}^{m}\left(t_{i}-x_{i}\right)^{2}} .
$$

Lemma 4.2. For $m \in \mathbb{N}$, $\mathbf{a}=\left(a_{0}, \ldots, a_{m}\right) \in \mathbb{R}^{m+1}, \mathbf{a}>-\mathbf{1}, \rho \geq 1, n \in \mathbb{N}$ and $\mathbf{x}=\left(x_{1}, \ldots, x_{m}\right) \in$ $\Delta_{m}$ :

i) $\mathbb{M}_{n}^{\rho, \mathbf{a}}\left(\mathbf{1}_{\Delta_{m}}\right)(\mathbf{x})=1$,

ii) $\mathbb{M}_{n}^{\rho, \mathbf{a}}\left(\operatorname{pr}_{i}\right)(\mathbf{x})=\frac{n \rho x_{i}+a_{i}+1}{\rho n+|\mathbf{a}|+m+1},(1 \leq i \leq m)$, 
iii) $\mathbb{M}_{n}^{\rho, \mathbf{a}}\left(\|\bullet-\mathbf{x}\|^{2}\right)(\mathbf{x})=\sum_{i=1}^{m} \frac{n \rho(\rho+1) x_{i}\left(1-x_{i}\right)+\lambda_{i}(\mathbf{a}, m, \mathbf{x})}{(\rho n+|\mathbf{a}|+m+1)(\rho n+|\mathbf{a}|+m+2)}$, where

$$
\lambda_{i}(\mathbf{a}, m, \mathbf{x}):=(|\mathbf{a}|+m+1)(|\mathbf{a}|+m+2) x_{i}^{2}-2(|\mathbf{a}|+m+2)\left(a_{i}+1\right) x_{i}+\left(a_{i}+1\right)\left(a_{i}+2\right) .
$$

Proof. For any $\mathbf{k} \in \Lambda, \mathbf{k}=\left(k_{0}, \ldots, k_{m}\right)$, we have

a) $F_{n, \mathbf{k}}^{\rho}\left(\mathbf{1}_{\Delta_{m}}\right)=1$;

b) $F_{n, \mathbf{k}}^{\rho}\left(\mathrm{pr}_{i}\right)=\frac{\rho k_{i}+a_{i}+1}{\rho n+|\mathbf{a}|+m+1},(1 \leq i \leq m)$;

c) $F_{n, \mathbf{k}}^{\rho}\left(\operatorname{pr}_{i}^{2}\right)=\frac{\left(\rho k_{i}+a_{i}+1\right)\left(\rho k_{i}+a_{i}+2\right)}{(\rho n+|\mathbf{a}|+m+1)(\rho n+|\mathbf{a}|+m+2)},(1 \leq i \leq m)$.

Then, we can apply the known results for Bernstein operator on a simplex.

By passing to limit $\mathbf{a} \rightarrow \mathbf{- 1}$ and using Lemma 4.2 and Theorem 3.1, we obtain:

Corollary 4.1. For $m \in \mathbb{N}, \rho \geq 1, n \in \mathbb{N}$ and $\mathbf{x}=\left(x_{1}, \ldots, x_{m}\right) \in \Delta_{m}$ :

i) $\mathbb{U}_{n}^{\rho}(\ell)=\ell$, for any affine function,

ii) $\mathbb{U}_{n}^{\rho}\left(\|\bullet-\mathbf{x}\|^{2}\right)(\mathbf{x})=\frac{\rho+1}{n \rho+1} \sum_{i=1}^{m} x_{i}\left(1-x_{i}\right)$.

Lemma 4.3. For $m \geq 2$, we have

$$
\max \left\{\sum_{i=1}^{m} x_{i}\left(1-x_{i}\right) \mid\left(x_{1}, \ldots, x_{m}\right) \in \Delta_{m}\right\}=\frac{m-1}{m} .
$$

Proof. We can apply for instance the Kuhn-Tucker conditions for this maximization problem and the optimum is obtained for $x_{i}=\frac{1}{m},(1 \leq i \leq m)$.

For $f \in C\left(\Delta_{m}\right), h>0$, define

$$
\omega_{1}(f, h)=\sup \left\{|f(\mathbf{x})-f(\mathbf{y})|, \mathbf{x}, \mathbf{y} \in \Delta_{m},\|\bar{x}-\bar{y}\| \leq h\right\} .
$$

Theorem 4.3. For $m \in \mathbb{N}, \mathbf{a}=\left(a_{0}, \ldots, a_{m}\right) \in \mathbb{R}^{m+1}, \mathbf{a}>-\mathbf{1}$, and $\rho \geq 1$, we have

$$
\left\|\mathbb{M}_{n}^{\rho, \mathbf{a}}(f)-f\right\| \leq 2 \omega_{1}\left(f, \sqrt{\mu_{n}}\right), f \in C\left(\Delta_{m}\right), n \in \mathbb{N},
$$

where

$$
\mu_{n}=\sup _{\mathbf{x} \in \Delta_{m}} \mathbb{M}_{n}^{\rho, \mathbf{a}}\left(\|\bullet-\mathbf{x}\|^{2}\right)(\mathbf{x})
$$

and

$$
\mu_{n}=\mathrm{O}\left(\frac{1}{n}\right) \text {, uniformly with regard to } \rho \in[1, \infty) .
$$

Proof. For $m \geq 2$, from Lemma 4.2, Lemma 4.3, since $|\mathbf{a}|+m+1>0$, it follows:

$$
\begin{aligned}
\mu_{n} & \leq \sum_{i=1}^{m} \frac{n \rho(\rho+1) x_{i}\left(1-x_{i}\right)+\left\|\lambda_{i}(\mathbf{a}, m, \bullet)\right\|}{(n \rho)^{2}} \\
& \leq \frac{1}{n}\left[\frac{\rho+1}{\rho} \cdot \frac{m-1}{m}+\frac{1}{n \rho^{2}} \sum_{i=1}^{m}\left\|\lambda_{i}(\mathbf{a}, m, \bullet)\right\|\right] \leq \frac{1}{n}\left(2+\sum_{i=1}^{m}\left\|\lambda_{i}(\mathbf{a}, m, \bullet)\right\|\right) .
\end{aligned}
$$

This final estimate exists also in the case $m=1$. Then, we apply the generalized theorem of Shisha and Mond, given in Altomare and Campiti [2]- Proposition 5.1.5. in the following form:

$$
|L(f)(\mathbf{x})-f(\mathbf{x})| \leq\left(1+\frac{1}{\delta^{2}}(L(e)(\mathbf{x})-e(\mathbf{x}))\right) \omega_{1}(f, \delta),
$$

where $L: C(K) \rightarrow B(K)$ is a positive linear operator which preserves affine functions, $K$ is a compact set in an inner product space, $e(\mathbf{x})=\|\mathbf{x}\|^{2}, \mathbf{x} \in K, f \in C(K)$ and $\delta>0$. Here, we take $K=\Delta_{m}, L=\mathbb{M}_{n}^{\rho, \mathbf{a}}$ and $\delta=\sqrt{\mu_{n}}$. 
Theorem 4.4. For $m \in \mathbb{N}$ and $\rho \geq 1$, we have

$$
\begin{aligned}
& \left|\mathbb{U}_{n}^{\rho}(f)(\mathbf{x})-f(\mathbf{x})\right| \leq 2 \omega_{1}\left(f, \sqrt{\frac{\rho+1}{n \rho+1} \sum_{i=1}^{m} x_{i}\left(1-x_{i}\right)}\right), f \in C\left(\Delta_{m}\right), n \in \mathbb{N}, \mathbf{x} \in \Delta_{m}, \\
& \left\|\mathbb{U}_{n}^{\rho}(f)-f\right\| \leq 2 \omega_{1}\left(f, \sqrt{\frac{\rho+1}{n \rho+1} \cdot \max \left\{\frac{1}{4}, \frac{m-1}{m}\right\}}\right), f \in C\left(\Delta_{m}\right), n \in \mathbb{N} .
\end{aligned}
$$

Proof. We apply Corollary 4.1, Lemma 4.3 and the generalized theorem of Shisha and Mond as in the proof of Theorem 4.3.

Corollary 4.2. For any $f \in C\left(\Delta_{m}\right)$, we have

i) $\lim _{n \rightarrow \infty}\left\|\mathbb{M}_{n}^{\rho, \mathbf{a}}(f)-f\right\|=0$, where $\mathbf{a}>-\mathbf{1}, \rho \geq 0$,

ii) $\lim _{n \rightarrow \infty}\left\|\mathbb{U}_{n}^{\rho}(f)-f\right\|=0$, where $\rho \geq 0, m \geq 2$.

Corollary 4.3. For any $m \in \mathbb{N}, \rho \geq 1$ and $n \in \mathbb{N}$, operator $\mathbb{U}_{n}^{\rho}$ interpolates each function $f \in C\left(\Delta_{m}\right)$ in the vertices of the simplex $\Delta_{m}$, i.e.,

$$
\mathbb{U}_{n}^{\rho}(f)\left(\mathbf{e}_{i}\right)=f\left(\mathbf{e}_{i}\right),(0 \leq i \leq m) .
$$

More refined estimates with second order moduli can be given for operators $\mathbb{U}_{n}^{\rho}$ because they reproduce the affine functions.

For $f \in C\left(\Delta_{m}, Y\right), h>0$, define

$$
\omega_{2}(f, h)=\sup \left\{\left|f(\mathbf{x})-2 f\left(\frac{1}{2}(\mathbf{x}+\mathbf{y})\right)+f(\mathbf{y})\right|, \mathbf{x}, \mathbf{y} \in \Delta_{m},\|\mathbf{x}-\mathbf{y}\|<h\right\} .
$$

We apply the following scalar version of a theorem given in [23, Th. 7.2.4].

Theorem A. Let $D \subset \mathbb{R}^{m}$ be a compact convex set. Let $F: C(D) \rightarrow \mathbb{R}$ be a functional given by a positive Borel measure $\mu$. Suppose $\mu(D)=1$. Let $\mathbf{x} \in D$ be the barycenter of $\mu$. Then

$$
|F(f)-f(\mathbf{x})| \leq\left[m+\frac{1}{2} h^{-2} F\left(\|\bullet-\mathbf{x}\|^{2}\right)\right] \omega_{2}(f, h)
$$

for $f \in C(D), h>0$.

Theorem 4.5. For $n \in \mathbb{N}, \rho>0, f \in C\left(\Delta_{m}\right), m \geq 2$ and $h>0$

$$
\left\|\mathbb{U}_{n}^{\rho}(f)-f\right\| \leq\left(m+\frac{1}{2 h^{2}} \frac{\rho+1}{\rho n+1} \cdot \frac{m-1}{m}\right) \omega_{2}(f, h) .
$$

Proof. For any fixed $\mathbf{x} \in \Delta_{m}$, define the functional on $C\left(\Delta_{m}\right), F(f)=\mathbb{U}_{n}^{\rho}(f, \mathbf{x})$. This is a functional defined by a positive Borel measure, say $\mu$. From Corollary $4.1-\mathrm{i})$, it follows that $\mathrm{x}$ is the barycenter of $\mu$. Then, we can apply Theorem A.

An other second modulus can be defined as follows. For $f \in C\left(\Delta_{m}\right)$ and $h>0$, define

$$
\begin{aligned}
\tilde{\omega}_{2}(f, h) & =\sup \left\{\left|\sum_{i=1}^{p} \lambda_{i} f\left(\mathbf{y}_{i}\right)-f(\mathbf{x})\right|, p \in \mathbb{N}, \mathbf{x}, \mathbf{y}_{i} \in \Delta_{m},\right. \\
\mathbf{x} & \left.=\sum_{i=1}^{p} \lambda_{i} \mathbf{y}_{i}, \lambda_{i} \in(0,1), \sum_{i=1}^{p} \lambda_{i}=1,\left\|\mathbf{x}-\mathbf{y}_{i}\right\| \leq h\right\} .
\end{aligned}
$$

The theorem below is a scalar version of a result given in [23, Th. 6.2.9]. 
Theorem B. Let $D \subset \mathbb{R}^{m}$ be a compact convex set. Let $F: C(D) \rightarrow \mathbb{R}$ be a functional given by a positive Borel measure $\mu$. Suppose $\mu(D)=1$. Let $\mathbf{x} \in D$ be the barycenter of $\mu$. Then

$$
|F(f)-f(\mathbf{x})| \leq\left[1+h^{-2} F\left(\|\bullet-\bar{x}\|^{2}\right)\right] \tilde{\omega}_{2}(f, h)
$$

for $f \in C(D)$ and $h>0$.

In a similar mode as in the proof of Theorem 4.5, we obtain

Theorem 4.6. For $n \in \mathbb{N}, \rho>0, f \in C\left(\Delta_{m}\right), m \in \mathbb{N}$ and $h>0$,

$$
\left\|\mathbb{U}_{n}^{\rho}(f)-f\right\| \leq\left(1+h^{-2} \frac{\rho+1}{\rho n+1} \cdot \max \left\{\frac{1}{4}, \frac{m-1}{m}\right\}\right) \tilde{\omega}_{2}(f, h) .
$$

\section{REFERENCES}

[1] T. Acar, A. Aral and I. Raşa: Modified Bernstein-Durrmeyer operators, Gen. Math., 22 (1) (2014), $27-41$.

[2] F. Altomare, M. Campiti: Korovkin-type approximation theory and its applications, Walter de Gruyter, Berlin-New York (1994).

[3] A. Attalienti: Generalized Bernstein-Durrmeyer operators and the associated limit semigroup, J. Approx. Theory, 99 (1999), 289-309.

[4] E. Berdysheva, K. Jetter: Multivariate Bernstein-Durrmeyer operators with arbitrary weight functions, J. Approx. Theory, 162 (2010), 576-598.

[5] H. Berens, Y. Xu: On Bernstein-Durrmeyer polynomials with Jacobi weights, Approximation Theory and Functional Analysis, (College Station, TX, 1990), 25-46, Academic Press, Boston (1991).

[6] W. Z. Chen: On the modified Bernstein-Durrmeyer operator, In Report of the Fifth Chinese Conference on Approximation Theory, Zhen Zhou, China (1987).

[7] M. M. Derriennic: Sur l'approximation de fonctions intégrables sur [0,1] par des polynômes de Bernstein modifies, J. Approx. Theory, 31 (1981), 325-343.

[8] M. M. Derriennic: On multivariate approximation by Bernstein-type polynomials, J. Approx. Theory, 45 (2) (1985), 155-166.

[9] Z. Ditzian: Multidimensional Jacobi-type Bernstein-Durrmeyer operators, Acta Sci. Math., (Szeged) 60 (1995), $225-243$.

[10] S. Durrmeyer: Une formule d'inversion de la transforme de Laplace: Application a la theorie de moments, Dissertation, These de 3e cycle, Faculté de Sci. de Univ. Paris, (1967).

[11] I. Gavrea: The approximation of the continuous functions by means of some linear positive operators, Result. Math., 30 (1-2) (1996), 55-66.

[12] H. Gonska, R. Păltănea: Simultaneous approximation by a class of Bernstein-Durrmeyer operators preserving linear functions, Czech. Math. J., 60 (135) (2010), 783-799.

[13] H. Gonska, I. Raşa and E.- D. Stănilă: The eigenstructure of operators linking the Bernstein and the genuine BernsteinDurrmeyer operators, Mediterr. J. Math., 11 (2014), 561-576.

[14] T. N. T. Goodman, A. Sharma: A modified Bernstein-Schoenberg operator, Proc. of the Conference on Constructive Theory of Functions, Varna (1987) (ed. by Bl. Sendov et al.). Sofia: Publ. House Bulg. Acad. of Sci., (1988), 166-173.

[15] V. Gupta, G. Tachev: Approximation with positive linear operators and linear combinations, Springer, (2017).

[16] B. Li: Approximation by a class of modified Bernstein-Durrmeyer operators, Approx. Th. Appl., 10 (1994), $32-44$.

[17] A. Lupaş: Die Folge der Betaoperatoren, Dissertation, Universität Stuttgart (1972).

[18] D. H. Mache, D.X. Zhou: Characterization theorems for the approximation by a family of operators, J. Approx. Theory, 84 (1996), 145-161.

[19] P. E. Parvanov, B. D. Popov: The limit case of Bernstein's operators with Jacobi weights, Math. Balkanica (N.S.), 8 (1994), 165-177.

[20] R. Păltănea: Sur un operateur polynômial défini sur l'ensemble des fonctions intégrables, Babeş Bolyai Univ., Fac. Math., Res. Semin., 2 (1983), 101-106.

[21] R. Păltănea: Une propriété d'extrémalité des valeurs propres des opérateurs polynômiaux de Durrmeyer généralisés, L'Analyse Numér. et la Theor. de l'Approx., 15 (1986), 57-64.

[22] R. Păltănea: On a limit operator. Proc. of the "Tiberiu Popoviciu" Itinerant Seminar of Functional Equations, Approximation and Convexity, (ed. by E. Popoviciu), Srima Press, Cluj-Napoca (2001), 169-179.

[23] R. Păltănea: Approximation Theory Using Positive Linear Operators, Birkhäuser, Boston (2004).

[24] R. Păltănea: A class of Durrmeyer type operators preserving linear functions, Ann. Tiberiu Popoviciu Semin. Funct. Equ. Approx. Convexity, 5 (2007), 109-117.

[25] R. Păltănea: Generalized Bernstein-Durrmeyer operators on a simplex, Gen. Math., 20 (5) (2012), 71-82. 
[26] T. Sauer: The genuine Bernstein-Durrmeyer operator on a simplex, Results. Math., 26 (1-2) (1994), 99-130.

[27] L. Song: Some approximation theorems for modified Bernstein-Durrmeyer operators, Approx. Th. Appl., 10 (1994), 1-12.

[28] S. Waldron: A generalised beta integral and the limit of the Bernstein-Durrmeyer operator with Jacobi weights, J. Approx. Theory, 122 (2003), 141-150.

RADU PĂLTĂNEA

TRANSILVANIA UNIVERSITY OF BRAŞOV

FACULTY OF MATHEMATICS AND COMPUTER SCIENCE

RO- 500091, BRAŞOV, ROMANIA

ORCID: 0000-0002-9923-4290

E-mail address: radupal tanea@yahoo.com 